\title{
Live Sketchnoting Across Platforms: Exploring the Potential and Limitations of Analogue and Digital Tools
}

\author{
Marina Fernández Camporro \\ UCL Interaction Centre \\ University College London \\ London, United Kingdom \\ marina.camporro.18@ucl.ac.uk
}

\author{
Nicolai Marquardt \\ UCL Interaction Centre \\ University College London \\ London, United Kingdom \\ n.marquardt@ucl.ac.uk
}

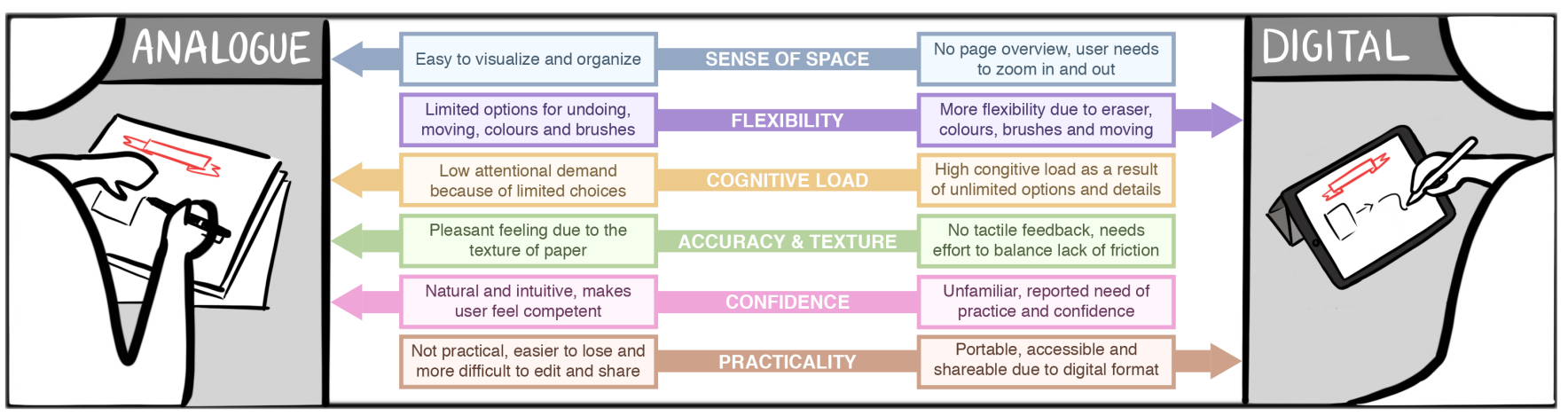

Figure 1. Overview of Study Findings: potential and limitations of analogue and digital sketching tools.

\section{ABSTRACT}

Sketchnoting is the process of creating a visual record with combined text and imagery of an event or presentation. Although analogue tools are still the most common method for sketchnoting, the use of digital tools is increasing. We conducted a study to better understand the current practices, techniques, compromises and opportunities of creating both pen\&paper and digital sketchnotes. Our research combines insights from semi-structured interviews with the findings from a within-subjects observational study where ten participants created real time sketchnotes of two video presentations on both paper and digital tablet. We report our key findings, categorised into six themes: insights into sense of space; trade-offs with flexibility; choice paradox and cognitive load; matters of perception, accuracy and texture; issues around confidence; and practicalities. We discuss those findings, the potential and limitations of different methods, and implications for the design of future digital sketchnoting tools.

\section{Author Keywords}

Sketchnoting; sketching; visual note taking; tablet; pen interaction; digital ink.
CSS Concepts

- Human-centered computing Human computer interaction (HCI); Interaction Techniques; HCI design and evaluation methods; User studies.

\section{INTRODUCTION}

Taking visual notes can improve recall and retention, enhance creativity, provide with a higher level of understanding and even result in better listening skills $[12,27]$. Dual coding, the idea that when we process concepts from verbal and visual channels at the same time we increase comprehension and recall, can be used to explain this technique's benefits [9]. In addition, we excel at remembering images. According to Perry and Weimar [32], we remember $10 \%$ of a piece of information we hear, but $65 \%$ if we add a picture.

One particular form of visual note taking are sketchnotes. This term was coined by Mike Rohde describing "rich visual notes created from a mix of handwriting, drawings, handdrawn typography, shapes, and visual elements like arrows, boxes and lines" [36]. They differ from common notes in their inclusion of visual imagery, focus on the main points rather than details, and a non-linear, more flexible layout [33]. They emphasize the content and ideas rather than the quality of the final representation [26], and are often drawn in real time to capture the essence of a talk, lecture, video or any other event.

Sketchnoting is a highly flexible and adaptable technique. Being able to customize notes, each person will use their own personal approach, which results in a better likelihood of processing, revisiting and following up the information [39]. The most common tools for creating sketchnotes are simply 
pen and paper, but tablet computers for digital sketching are becoming increasingly popular [12]. We were interested in better understanding the process of creating both analogue and digital live-drawn sketchnotes, building and extending on related studies about the use and limitations of digital styli [1], digital sketching of design renderings [14], and hybrid use of pen and touch interaction [21].

In particular, we designed a study to directly compare analogue and digital live sketchnoting. This research was motivated by Riche et al.'s [35] diary study and survey about everyday analogue pen use to inform the design of digital inking tools, specifically their analysis of affordances of analogue and digital pens. The methodology of our experiment was informed, among others, by the experimental setup and study design by Annett et al. [1] and Mueller and Oppenheimer [27]. The focus of our study was to have 10 participants create live analogue and digital sketchnotes of two video talks, followed up by semistructured interviews. This allowed us to explore the different techniques and experiences when using tablet with digital pen and analogue pen with paper for creating sketchnotes (Figure 1). We were able to investigate the potential and limitations of analogue and digital tools in order to inform the design of note-taking applications, platforms and systems - either directly for sketchnoting practice, or sketching more generally $[19,44]$.

With our detailed analysis, we contribute a number of findings about the use of analogue and digital tools for live sketchnoting, categorised into six themes: (1) insights into people's sense of space; (2) trade-offs with flexibility of available tools; (3) discussion of the choice paradox and cognitive load; (4) matters of perception, accuracy and texture; (5) issues around confidence when using tools; and (6) practicalities of different media. We close our paper with a discussion of these findings and their relevance for the design of digital sketching-related tools. Throughout these themes, some of our findings confirm results from earlier research, whereas others are bringing up novel insights, such as how affordances of digital and analogue tools might fit different stages of the sketchnote creation process.

\section{CHARACTERISTICS OF SKETCHNOTES}

In this section, we briefly synthesize the key characteristics of what makes a sketchnote.

A sketchnote is a one-page, memorable document that includes visual elements and text to innovatively make the recorded information more meaningful and engaging $[12,28]$. There are certain elements that, in combination, are distinctive of a sketchnote (Figure 2). Text in sketchnotes is hand-written, and different fonts and colours set the tone and relative importance of the idea [9,11]. Simple sketches are among the most representative elements of a sketchnote. They are used to visualize information and to maximize efficiency and the impact of ideas [12,30]. Connectors, such as arrows and bullets, together with frames and boxes, which act as containers and dividers, conform a structure, make the content flow and enhance visual hierarchy $[29,43]$. They help to display a clear narrative. Colours and shades are used in sketchnotes to emphasize, but the most common colour scheme is monochrome for its simplicity [43]. Lastly, layouts in sketchnotes tend to be flexible and non-linear [33], and they may be geometrical or random [12].

Besides the explained elements, there are properties that are intrinsic to sketchnoting. Sketchnotes can be recorded live or they can be used to redo traditional notes [30]. In addition, the technique can be used to summarize books, movies, publications, travel plans, and brainstorming sessions, among others [12]. In the scope of this research, we focus on sketchnotes done in real time, for example to summarise talks at conferences, lectures or presentations. Although the process varies individually, the sketchnoter will commonly start by simple planning of the structure, adding the title, description and other details, such as a speaker's portrait or their past presentations (preparation stage). Then they will record the main concepts and add more levels of detail. Lastly, in the editing stage, the sketchnote will be refined to make the visual hierarchy more salient [13].

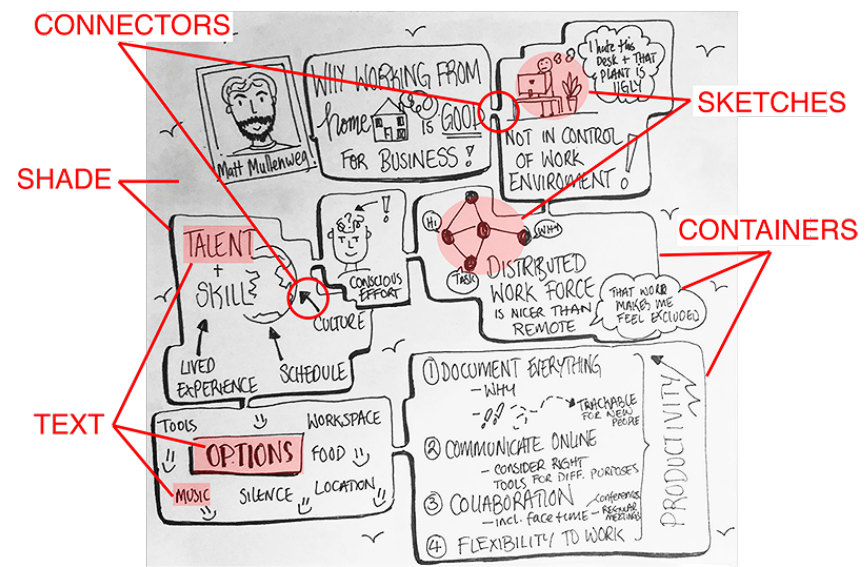

Figure 2. Elements of a Sketchnote (the sketchnote shown was drawn by a participant of our study).

Given its basis on simple and easy to understand visuals, sketchnoting is appropriate for both skilled drawers and beginners; no prior sketching knowledge is required [28,29]. The sketchnote is generally messy and might contain mistakes. It is about ideas, not art. Real time sketchnoting involves simultaneously listening, processing, synthesizing, writing and drawing [11,12,28]. Sketchnoting can potentially enhance idea generation, creative and sketch confidence, design ability and the learning process [28]. Sketchnotes are very personal, which may result in other people not being able to immediately understand someone else's sketchnote [12]. However, there is an increasing trend of sharing sketchnotes on social media, which allows their creator to connect with the speaker and attendees, as well as non-attendees $[30,43,47]$. 
For creating sketchnotes, pen and paper (usually a pen, a marker and a bound sketchbook) are the most common tools and material. Digital platforms are increasingly popular for sketchnoting $[12,43]$, for example using Apple iPad or Microsoft Surface with the digital pens. Because of our focus on both digital and analogue sketching, in the next section we synthesise key findings from related work on studies focusing on digital and analogue techniques for sketching and note-taking.

\section{BACKGROUND: ANALOGUE VS. DIGITAL NOTE TAKING} Digital pens as additional input method on tablet computers have the potential to complement (multi-)touch interaction. Although their accuracy has improved, there are still obstacles to overcome in their adoption [3,35] - for instance, over half of the people who buy a digital pen for inking use it less than expected at first [35]. Paper is usually the baseline to compare the digital experience with [1], and by better understanding interaction with analogue tools and by comparing analogue to digital pen use we can inform the design of digital tools [7].

\section{Issues and Limitations of Digital Inking}

Latency and accuracy: The most frequent issues found when studying interactions with a digital pen were impact latency, stylus inaccuracy, lack of intended input and unintended touch $[1,2,14,35]$. These limitations may result in stray ink strokes and unintentional interaction, which cause frustration on the user and might result in them avoiding the platform.

Lack of tactile feedback is another prominent issue. The mismatch of friction between the drawing/writing surface (usually the glass of the display) and the pen caused the soft texture to not seem natural and people have different expectations for the pen "feel" $[1,35]$. A related issue is the very limited choice of different shapes and weights for digital pens [1], making them feel impersonal. Moreover, grip and hand movements were reported to be different from analogue tools [3,35]. Users needed to learn new ways of interacting.

Expressiveness and cognitive load: Digital tools still do not match the diversity of artistic styles found with physical methods, even considering the improvements in stroke rendering and beautification [48]. Additionally, digital tools use modes to mimic physical tools, which can lead to increased cognitive load. Shilman et al. [40] discovered that users are reluctant to switch modes to avoid getting distracted. For example, even though digital tools provide a functioning eraser tool, people often simply scribbled out their errors. Digital tools consume more attention and resources because of the abstract mappings the user needs to remember [45]. Users choose not to use novel functions like asymmetric bimanual interaction due to lack of familiarity with them [46].

In addition, some have claimed that the quality and choice availability of the digital tools result in the temptation of adding more detail than needed, slowing the process and constraining the free, non-judgmental ideation found on paper [25]. This is why analogue sketching is often associated with the rapid ideation process and drafting, whereas digital inking has been linked to the detailed refinement process or crafting $[4,35]$. This is in line with research that showed that paper-based sketches were better than digital tools for synthesizing, problem-solving, ideating and communicating ideas [14,41,42].

Practicalities: On top of these limitations, there were issues that emerged related to the practicality of digital tools that were identified as key reasons to not use them. Users reportedly disliked the weight, depending on battery and availability, as well as the lack of immediacy given the need to find and launch a particular app [35].

\section{Potential and Advantages of Digital Inking}

On the other hand, the development of digital styli for tablet brings many advantages, such as the ability to annotate digital content in its own context [35]. Contrary to traditional digital tools (touch, mouse and keyboard), the digital pen has allowed to combine hand-written text, imagery and diagrams - which is essential for visual note taking and provides rich personal expressiveness [34].

Flexibility has been highlighted as digital tools' biggest advantage. This is achieved by the option to change the style and colour of the strokes, and allowing for dynamic editing and easy correcting of mistakes or going back, making the user experience more adaptable [45]. The "undo" function was found to be one of the most important ones, resulting in greater confidence $[14,48]$. Another feature that increases flexibility is the option to copy, paste and move things around $[40,48]$. These functions enable the user to develop and perfect the layout in the editing stage, as opposed to having to plan it beforehand. Tsandilas et al. [48] also identified the ability to zoom in and out to refine details as a strength. On top of these, the option to change between canvases and layers also added to flexibility [45].

Decreased friction: Due to the lack of friction on the surface, some studies found that inking was faster on tablet than paper $[14,17,48]$. This means that the user needs to apply additional control on their movements, which could have a learning curve. However, a study by Gerth et al. [16] showed that users were capable of adapting, and if successful, inking would be easier and faster on tablet than on paper. Contrary to what was previously stated, recent research has found that users perceived digital tools to be more accurate than analogue tools [1,14], which might reflect recent technological improvements.

Metaphors: The digital experience is often enhanced with the use of metaphors by making it more physical-like, and therefore more natural and familiar [45]. An example of this can be found in reproducing physical ink dynamics like thickness and path smoothing using speed and pressure [1]. In contrast, some digital-only features are also introduced, such as using bimanual interaction to divide tasks $[1,21,22]$. 
This builds on the user's preference to switch modes and activate buttons with their non-preferred hand, which results in faster performance [24]. However, if users do not learn the gestures and combinations, this feature could add unnecessary cognitive load and result in them avoiding mode switching.

Other features that have been identified as positive in previous research were the ability to search [40], screen and stylus aesthetics and stroke beautification [1]. When it comes to practical strengths, having everything in one place, being able to access other media and upload the final versions to the Internet were highlighted. This is due to their connectivity and portability, which make them ideal for productivity purposes [1,25].

\section{The Analogue "vs." Digital Debate}

There is an ongoing debate about the comparison of digital vs. analogue tools for writing and sketching. For the digital to become successful, it has to support and enhance, but not limit or detract from the core analogue experience [4]. However, there are certain characteristics that make the analogue unique, and it might be impossible for digital tools to achieve them. For example, the permanence of errors, which are said to make each piece of work uniquely imperfect and irreproducible [6]. To illustrate this, one of the participants in Macdonald's research [25], after using an iPad, claimed that it lacked "the scars of the thinking process." These "scars" or errors symbolize learning points and mark an authentic involvement with the medium.

There has been a tendency for participants to prefer analogue tools to their digital counterparts [1,7]. Sellen and Harper [38] found that in work environments, digital tools were not advanced enough to offer a suitable alternative, so people chose to use paper instead. Although that research is from around two decades ago, attitudes towards the digital tend to remain similar [7]. However, when it comes to choosing between the two, some have claimed that posing the question of deciding for one or the other is irrelevant. Each offers a different type of experience, their own strengths and weaknesses and they should complement each other instead of being mutually exclusive [31]. The choice may vary individually and depend on the availability and nature of the task at hand [25]. With this research we aim understand the nuances of use with each platform, to potentially enhance the interaction in the future.

\section{METHOD}

In order to explore the potential and limitations of analogue and digital tools for live sketchnoting, we conducted a study in two parts: first, an observational study with the aim to compare the strategies used with both digital and analogue tools; and second, in-depth interviews analysed the experience and attitudes towards the different platforms.

\section{Participants}

Ten participants (eight female) were recruited for the study. Inclusion criteria were being familiar with sketchnoting and being over 18 years old. It was not required to be an experienced sketchnoter or to regularly use digital tools. Nine out of ten participants reported nine to twelve months of sketchnoting experience, and the remaining one reported seven years. All participants were familiar with analogue sketching tools but only one used digital tools for sketchnoting. Three other participants were familiar with inking on tablet, but only used it for other purposes. Except for one participant, all of them had comparable sketchnoting experience, and their first time using a digital tablet for that purpose was during our study. Participants were recruited through posts on social media (Meetup, Twitter and Slack), handing flyers in one of the SketchnoteLDN meetups and at University College London. Each participant was rewarded a $£ 8$ Amazon voucher.

\section{Materials}

A black gel pen, a black sharpie, pencil, eraser, several coloured markers and pens and A4 sized paper were provided for the first part of the experiment. Some participants chose to use their own pens. The digital tool used in the second study part was an iPad Pro 9.7-inch with the compatible Apple Pencil, and the drawing software Notability and Procreate pre-installed. Notability was the default application (due to its simplicity and being easy to learn) to be used in the experiment, but participants were allowed to use another application if they voiced their preference (only one participant used the more advanced Procreate). The functionalities used were two available brushes, different thicknesses and colours, the eraser, undo function and the "scissors" tool, which allows to select, copy, paste, and move around. The experiments were filmed using a tripod and an iPhone 8 (Figure 3 ).

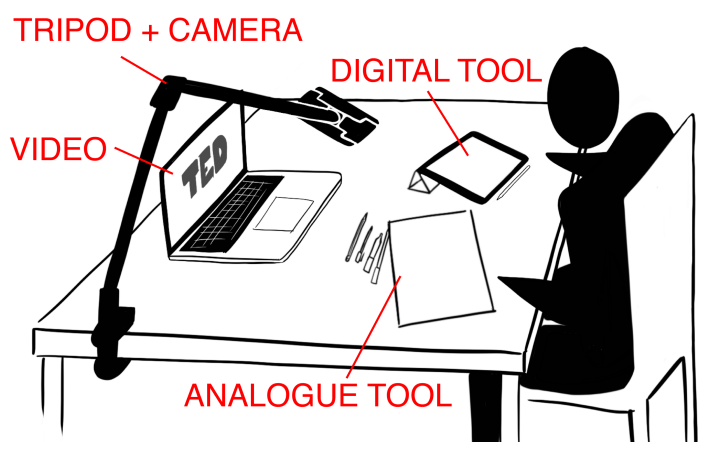

Figure 3. Study Setup.

The two videos to sketchnote were pre-recorded talks from the TED website. English subtitles were switched on. Two videos were chosen in order to alternate: "3 psychological tricks to help you save money" by Wendy de la Rosa ( 5 minutes) and "Why working from home is good for business" by Matt Mullenweg ( 4 minutes).

\section{Design}

The observational study was within-subjects. The independent or changing variable was the platform used. All participants used both analogue and digital tools. Half the 
participants were asked to sketchnote Video 1 on paper and Video 2 on iPad, and vice versa. This was done to counter balance systematic differences caused by the videos [23]. Observations were followed by semi-structured interviews. The study has been approved by the University College London Ethics Board.

\section{Procedure}

The two parts of the study were carried out with each participant individually. The full study took approximately 45 minutes per participant. The participant was greeted, explained the aim of the study and asked to read and sign the information sheet and consent form. Before the observational study, participants were asked how long they had been familiar with sketchnoting and what their preferred platform was for that purpose.

Observational Study Part 1: Analogue Tools. To start the study, the participant was told to use any pens or markers they preferred or to use their own. They were given as much time as needed for the preparation stage, in which they would write down the title and name of the speaker, and sometimes a sketch of the presenter. After the participant notified that they were ready to start live sketchnoting the talk, the video would be started and participants began sketching. After the video finished, the editing phase allowed participants to edit and embellish, add content, colours, layout, etc. after the end of the talk.

Observational Study Part 2: Digital Tools. Participants were introduced to the use of the Notability sketching application. They were also asked to use the application freely until they felt confident enough to use it in a live sketchnote. This part took maximum ten minutes. The rest of the study was conducted following the exact same procedure as in the first part.

At the end, we conducted semi-structured interviews. Participants were asked questions on their use of tools and overall experience, and follow-up questions were added for participants to elaborate on particular aspects of the sketching or techniques/strategies they used during part 1 and 2 of the study.

\section{Analysis}

For the observational study, we took field notes in real time for each of the participants. The video recordings were coded and analysed to complement the data collected in the observational notes. The recorded audio from the interviews was transcribed manually for each participant. To analyse the transcripts, a thematic analysis [18] was conducted using a bottom-up approach [5], following the six steps introduced by Braun and Clarke [8]. After familiarisation with the data, over 50 initial codes were generated. Initial coding was followed by an early search of themes and consequent classification. Thirteen themes were initially identified. These themes were later reviewed, defined and named. The final set consisted of six main themes (Figure 1). The early codes generated from the video annotations were later classified into categories corresponding to the final themes.

\section{FINDINGS}

Our findings section is divided into six parts, each corresponding to one of the final themes (see Figure 1 for a summary of all six themes).

\section{Theme 1: Sense of Space}

During the interviews, nine out of ten participants reported issues with getting a sense of the available space when using the digital tablet. They pointed out that paper was better at providing an overview. For instance, P3 stated that paper was better "because I can visualize everything I'm doing at the same time." They highlighted how knowing how much space there was left made it easier for them to plan their sketchnote: "because I can see it all [...] I know how much the space that I have, I can do the organization much better than in iPad" (P1). Some claimed that this issue might be due to the size of the iPad, which was smaller than the intended A4 size. P7, for instance, pointed out that "you're constrained by the size of the tablet and on paper you can have more space."

To solve this issue, digital tools offer the option to zoom in and out to understand the space better, and the resizing tool to adjust the elements and move them around the page. However, this alternative was often seen as an ineffective solution. As P6 stated, "I don't want to be wasting time resizing things." Participants mentioned in particular that these commands could not replace the natural constraint offered by paper:

"I know you can zoom in and out of this [iPad] and see the whole page, but it's not quite the same effect as literally having the page in front of you [...] and you don't have to move around to zoom in and out. (P3)

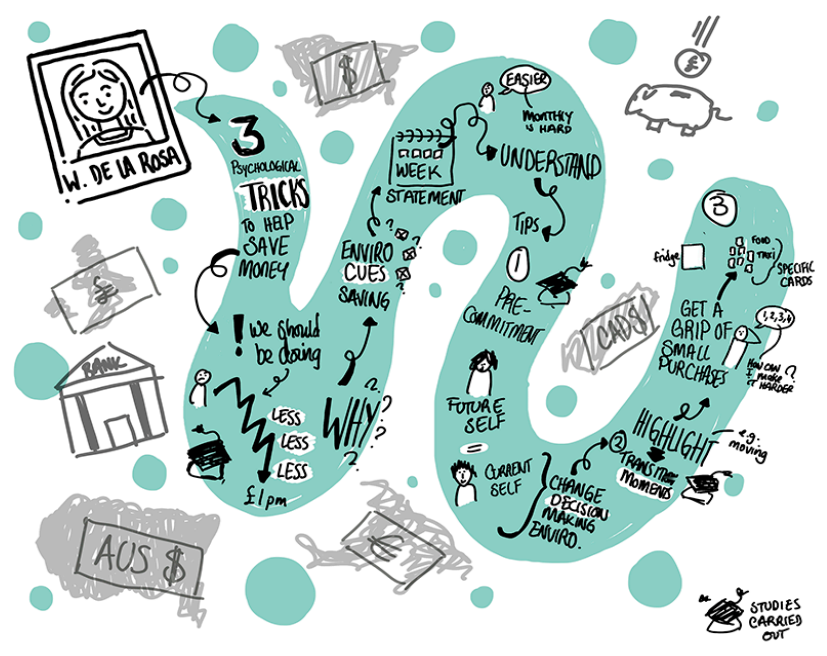

Figure 4. Path (green shape in the background) in P6's digital sketchnote. 
This issue of a missing sense of space was also noticeable when looking at the sketchnoting outcomes. Participants would often fill the whole page when working with pen\&paper, but either not use the entire page or go over its limits when working digitally. This was reflected on the proportions of the final documents.

One of the participants (P6) had developed a technique to overcome this limitation, which consisted in creating a coloured path as a guide beforehand and limit themselves to following it (Figure 4). This would allow them to get a sense of the space and how much there was left. P6 explained the rationale: "I would lose sense of the size of the page, and how much I can fill, [...] so having this path, I know I need to follow it." They emphasised that "on paper I don't need to do that, cause I can see the full size of the page."

During the analogue part of the study, a specific behaviour was noticed about the roles of hands with all participants: while sketching or writing, people would use their nondominant hand to fix the paper and adjust it to the part of the page where they were inking (Figure 5). This corresponds to earlier findings by Guiard [20] about the asymmetric roles of the dominant vs. non-dominant hands. We did not observe the same behaviour when participants used the digital tablet, and their non-dominant hand was kept unused most of the time.

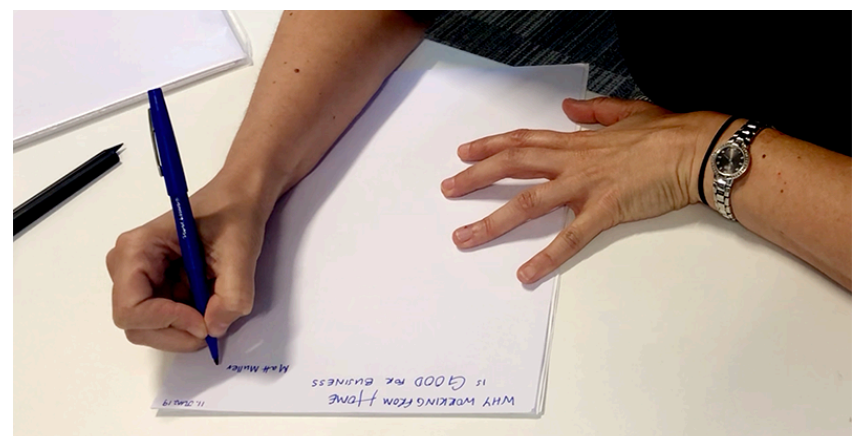

Figure 5. Non-dominant hand being used to hold (and often move and rotate) the page.

Theme 2: Flexibility

A key theme that emerged across all participants was flexibility. With the digital tablet, this was spread through different tools and commands, including undo, the eraser, the possibility to resize and move, the different colours and brushes and the wide range of options.

"It is much more flexible and the different tools are available much quicker and much more easily [...] with two clicks you have a different colour and a different style of pen and you have, you can erase, you can highlight, you can do fine lines, thick lines, everything [...] not have to worry about wasting paper or about like, something that can't be undone." (P9)
Undo

The undo and eraser tools were widely mentioned in the interviews and repeatedly observed in the study. For example, some participants mentioned that "I can always go back [...] I can make mistakes and I can edit it" (P5). This was contrasted to analogue tools, saying that "if you make a mistake you can't go back" (P2) and "on paper you can't erase as easily" (P8). Some participants said that because mistakes on paper were more permanent, they would be forced to start again in a new page: "I might do it again on another paper and redraw it [...] I cannot change it and, if it is ugly it's ugly" (P10).

One of the participants (P9), however, pointed out that not being able to easily erase on paper (in particular when using pens and markers instead of pencils) might be an advantage, because "it is good as something that forces you [...] to think about it [...] and that can teach you something".

\section{Resize and Move}

Another feature that was mentioned often was that of resizing and the ability to move things around. Several participants highlighted it as an advantage exclusive of the digital tablet, claiming that "what I like of the iPad is that I could like, resize stuff and move things around" (P4). Focus on this tool was mostly made in the editing stage to fix mistakes. For example, P7 claimed that "I can work on it later if I wasn't like, happy with it, I can like, improve on it." Emphasis was made on using these tools to work on the organization and layout: "cause I don't know what the speaker's going to say next, so it's kind of hard for me to plan the layout [...] If I do this on iPad, I can adjust the layout later" (P10) and "it makes it clearer to then edit stuff and show priorities in the talk" (P4).

\section{Colours and Other Tools}

A large number of options for colours, thicknesses and brushes was mentioned repeatedly in the interviews. In sketchnotes, these elements are used in particular to emphasize and highlight: "it's easier to make anything stand out. Because you can put like, really bright colours that are really thick colours, compared to diluted colours" (P8). P8 also used thicknesses to establish relationships, saying that "it makes it more standardized [...] you know these are related because they all look the same thickness or borders." Colours were also used for that goal: "I used different colours to separate the experiments [...] so it's really clear for me to see it" (P10).

The speed and accessibility of these tools, as opposed to analogue pens, was found to be particularly relevant by some participants, like P2, who claimed that "I really liked [...] not having to move things up and put them down. It's all just there in front of you" and P10, who stated that "you can change different pens and colours really quickly." In addition, P7 also pointed out the ability to adjust the strokes afterwards, saying that "you can manipulate the lines, you 
can change the lines [...] if you didn't really like that stroke you can increase it or decrease it."

\section{Theme 3: Choice Paradox and Cognitive Load}

Participants comments about flexibility and digital tools were often in contrast to the actual use of tools we observed. For most of the sketchnotes participants did not end up using many diverse colours or pens. Instead, often participants would use the same coloured pen on paper, or colour and brush thickness on iPad for the whole live sketchnote. They would add colours and refine details in the editing stage, but they would rarely change pens or brushes while taking the notes. In most cases, as observed in the final pieces of work, the analogue and digital sketchnotes were completed in the same style, using a similar variety of colours regardless of the platform.

Flexibility was most times referred to as an advantage of the digital tools, with statements such as "there was a lot of possibility on the iPad" (P3). However, in many cases, participants mentioned their concerns about having so many choices: "as it was quite a time-constrained activity, it felt like it was easier to just kind of pick one or two things and get on it" (P3). This relates to the well-known paradox of choice, referring to the situation in which less choices are better than many, since too many options increase anxiety and reduce happiness [37].

Consequently, participants voiced their preference towards a scenario with less options, saying that "sometimes it's easier just having, I know you gave me lots of options, but just having one pen [...] Just to say it's simpler, I guess" (P8). This preference was attributed to having to put less cognitive effort in choosing. For example, P4 claimed that on paper "you don't need to like, spend so much thought on selecting the right tool before you actually start." In addition, P8 highlighted how familiarity and haptic feedback helped to reduce cognitive load: "you can just stick to it and then you can alter the thicknesses just by eye by feeling it more and stuff. It is kind of harder in one way but also kind of more natural in another way."

It was found that digital sketchnotes would take longer to create due to time spent on fixing details and looking for perfection. For instance, P5 claimed that on digital, "probably I'll end up using more time because when I'm making marks or writing on paper, I know this is exactly what I want." For some participants, like P6, this would be a reason to avoid digital tools: "I don't do digital very often simply because for me, it is quite time consuming [..] you end up looking for perfection."

Some participants voiced their struggle with managing their cognitive load and focusing on what is most important when using digital tools. For example, P3 explains that "when I was doing it on paper I was more focused on the actual information whereas, when I was doing it on iPad I was thinking more about the aesthetics." Focusing their attention on the technology, and consequently not being able to follow the talk as well, made some participants aware of how much content was being captured: "the reason I get so mad with my digital is because I know I capture less. There's more content on paper because, for me, digital takes a bit longer and I'm more conscious of that" (P6). This was indeed visible in the resulting sketchnote for P6, where the difference in content captured was perceivable. However, this was not noticeable for the other participants.

There was another related observation. Several participants mentioned in the interview that they kept trying to make the digital as similar as paper as possible by mimicking the strategies used on paper when using the digital tablet. For example, in two cases, the participants would make a line thicker by going over it a couple times, just like it would be done when using a marker on paper, instead of changing the mode or thickness of the pen.

\section{Theme 4: Perception, Accuracy and Texture}

There were mixed opinions on the differences in speed between analogue and digital. Although some, like P2, claimed that the tablet was efficient, saying that "it sort of felt faster, I had more time to draw images," others stated the opposite, claiming that "on paper... I can do it really fast" (P10). We got similar mixed responses about the quality and aesthetics of the handwriting: P10 said that "the calligraphy on iPad is not really good" whereas others, like P7, claimed that "my sketching and my handwriting is not that nicely looking on paper as it is on the tablet."

Some participants highlighted the iPad's sensitivity for pen input, saying it was greater than when sketching on paper. For example, P1 even stated that it was too precise, saying that "it is quite accurate. But sometimes it might be too accurate. I cannot follow it." The reason why accuracy was not perceived as a good thing was likely due to the lack of friction. The technology was advanced and did not cause frustration due to latency or inaccuracy, but the glossy texture was unnatural and forced the participant to add extra control. As P6 pointed out: "it is not as neat, cause it is so slippery; that bugs me."

Moreover, participants reported positive attitudes towards the feeling of paper and the friction: "the feeling of writing on paper is very nice" (P9) and "you can feel the texture of the paper more" (P8). It was also emphasised how feeling the different pens provided with valuable haptic feedback, and lack of it in the tablet made the pen seem impersonal. P3, for instance, stated that "I like that on the different pens, you can sense different thicknesses on the paper whereas on this [iPad], obviously you can change it but the physical tool remains the same." Several participants pointed out that instead of it being a clear disadvantage, the glossy screen was just something that the user could and had to get used to. For example, P9 said that "some people might like the feeling of the screen, but I think it's like, okay, you definitely have to get used to it." 
Accidental movements and commands were observed with a few participants. In addition, in several cases, we noticed constant use of the zoom-in function to write and add details and the use of thicker lines than those in analogue pens. Although the edited elements were not particularly small, participants kept zooming in for editing and writing.

\section{Theme 5: Confidence}

When asked what is most positive when sketchnoting on paper, a considerable number of participants mentioned the familiarity and confidence provided by a platform they were experienced with. For example, P6 mentioned that "I feel like I'm more free on paper [...] I'm less confident when I work digitally." The feeling of familiarity caused participants to feel more capable and efficient, making them prefer paper: "I definitely felt more capable doing it on paper [...] I felt more competent with it" (P3). However, since these opinions were to a great extent, due to lack of practice, several participants suggested that they might like the digital tablet better if they were to get used to it and practice more: "it was the first time [...] I think I need a lot of practice but I did really enjoy the feeling of it" (P2).

\section{Theme 6: Practicalities}

Other aspects from our observations and interviews covered the practical aspects of the digital tablet, as opposed to paper. These mostly referred to the portability of the digital format. Being able to store everything organized in one place made the digital more convenient. As P4 claimed, "I can take it [iPad] everywhere with me and I have a lot of notebooks and papers and stuff, and it's really hard to search that and find the right stuff, so I think on iPad this is a lot easier." Similarly, paper sketchnotes were identified as easier to lose: "you have many sheets of paper in the end and you can lose it quite easily" (P9). In addition, $\mathrm{P} 10$ pointed out that "I can check it on computer, iPad, my phone... anywhere." This statement highlighted the accessibility of the digital format. P6 added that another convenient advantage of the digital format was the possibility of photo tracing, claiming that "I take photographs of something and then draw on top of it."

Working directly on the digital format also resulted in reduced paper waste and greater shareability due to easy connectivity. As P5 mentioned, "even if you do things on paper, you are likely to put it on digital to share [...] you might as well cut the step one and just go straight on to digital." However, the digital was also found to present disadvantages, such as potential eye strain from the screen: "over time your eyes will be more strained I think than working on paper" (P9). On top of that, another inconvenience that was emphasized by $\mathrm{P} 4$ was that, unlike in the study setup, where the iPad was over a table, "the iPad is a bit heavy and if you have to balance it [...] like in a conference and have it on your lap it's tough."

\section{Overall Preference}

Seven out of the ten participants, when asked which platform they preferred after the study, claimed that they would rather use the digital tablet for sketchnoting. Reasons given for this choice were attributed to the possibility to change the layout afterwards (P4), the ease to make improvements on the digital format (P7) or its flexibility and variety (P8). Some of the participants pointed out that they might prefer the tablet more after further practicing, like P5, who mentioned that "for sketchnoting, definitely if I get used to digital, then digital." One participant claimed that they would choose a different platform depending on the task at hand, distinguishing between pleasure and practicality: "personally, for note-taking, I prefer digital [...] I'd opt for the iPad simply because it is more flexible [...] but if I really want to enjoy drawing, I'd use the paper" (P9).

\section{DISCUSSION AND IMPLICATIONS}

In this last section, we will discuss our findings and point out implications for design and directions for future research.

\section{Discussion Areas}

Digital mimic analogue. The desire to reduce their cognitive load was emphasised by our participants attempts to mimic on the digital tablet the same techniques used on paper. This has been classified as an intuitive way of learning, by inferring new ideas from similar, familiar situations [10]. Several participants admitted to trying to make the digital " as normal" as possible in order to feel more confident when using it, easing the learning curve. This is why techniques specific to digital tools, such as frequent mode switches were actively avoided. As Bellamy et al. [4] pointed out, to succeed, digital tools need to extend the analogue interaction and not distract from it. In the context of live visual note taking, this is critical due to fact that users need to focus most of their attention on actively listening to the talk or presentation, and at the same time, decide what to ink and how to create their visual representation.

How accurate is too accurate? When it comes to the accuracy and texture of digital tools, latency, inaccuracy and unintended touch were previously identified as a common limitations of digital tablets $[1,3,14,35]$. However, we did not find these as major issues in our study, possibly due to recent technological improvements. In fact, few of the participants criticised that the digital tablet might be too accurate and more sensitive than paper. The fact that this was seen as a limitation might be due to the lack of friction. As mentioned earlier, Gerth's studies $[16,17]$ pointed out that even though this might make handwriting more difficult, users managed to get used to it, and once the behaviour was adapted, they could write faster than on paper. Literature emphasized the absence of tactile feedback due to the lack of friction and the impersonality of the digital pen [1], which was supported by our study where several participants complained about the lack of feedback.

Strengths of digital tools. Several practical aspects of digital tools were mentioned. Connectivity and portability were complemented by the benefits of the digital format, which remained a prevalent theme in the interviews. Another 
relevant conversation revolved around the overall preferences. Although previous research had shown that users overall preferred analogue tools [1,7,38], and the findings from this study would suggest the same, when asked about it, seven out of ten participants claimed to prefer the digital to the analogue for sketchnoting. This result, although not necessarily significant due to the reduced sample size, insinuates that even though the advantages of paper are more numerous, the ones found in digital tools outweigh them anyway in this context. This implies the possibility that the shift in attitudes is due to the technological improvements of digital tools, and could also be influenced by the study's specific focus on sketchnoting.

\section{Implications for Design}

Making sense of space. Some of our findings relate directly to earlier studies about using digital tools. Sense of space had already been identified as a possible limitation of digital tools [14]. The lack of overview and control and the need to zoom in and out frequently were identified in earlier studies as one of the most mentioned reasons to use paper over digital tablet, and aligns also with our observations and comments during the interviews. For the design of future digital note taking tools it will be interesting to investigate light-weight, rapid techniques to give a person the sense and control over the sketching space. For example, this could include easy to apply constraints of the (often) infinite drawing canvas of digital sketching tools, or techniques to more easily see an overview of the whole page without the need to use zoom-in/-out controls requiring to adjust the view (e.g., a snap-to-overview button that while pressing shows the whole page and then immediately jumps back to the zoomed-in view when released).

Flexibility vs simplicity: Flexibility, as the ability to choose between colours, stroke styles and sizes, go back and erase and move things around, is a key advantage of digital tools. As shown in the results, participants expressed their enthusiasm about having many possibilities available. However, this was in contrast to our third observation theme - that fewer options might be preferred by participants for note-taking. There is often a conflict between flexibility vs. simplicity. Participants questioned the actual usefulness of so much flexibility, with someone even claiming that not being able to erase mistakes could be preferable, similar to findings in Macdonald's study [25]. Previous research had stated that such flexibility resulted in the temptation of adding too much detail, making digital tools consume more attention than their analogue counterparts $[25,45]$, which we also confirmed in our interviews and observations. And even though they praised flexibility, participants claimed to prefer having less options to choose from in particular during the live sketchnoting phase. However, our findings also showed that in the post-processing phase (after the live sketchnoting was over), many participants would choose a much wider variety of digital tools and colours, mostly to apply emphasis and structure to the existing sketch. While current digital tools are mostly designed for either simplicity (e.g., Paper by 53) or flexibility with lots of choices (e.g., Procreate), an interesting future direction to investigate would be how digital tools can be better designed to support different phases of visual note taking - where we design applications differently for live sketchnoting vs. post processing vs. preparation before.

Towards digital drafting tools. The lower attentional demand of analogue tools can be related to the focus on information rather than aesthetics, which makes the communication of ideas more effective [14]. It can also be linked to the perception of analogue tools as "drafting" tools and digital tools as "crafting" tools $[4,35,41,42]$. The "limited" nature of analogue tools makes them more suitable for free ideation, whereas the flexibility of the digital is a good fit for refining and adding detail. A possible focus for future designs of digital tools could then be the exploration how digital tools could behave and function more like rapid analogue drafting tools. This might include very strict constraints on the number of available tools and options.

The role of the non-dominant hand. Interestingly, with pen and paper we observed participants' asymmetric roles of the dominant vs. non-dominant hands [20], but none of the participants moved the tablet while interacting. It is unclear why this is: one possible reason is likely the heavier weight of the tablet and increased friction on the table surface, or maybe that the availability of digital tools such as zoomin/out might make the bi-manual change of tablet orientation or position seem redundant to participants. Given that one of Guiard's [20] findings was that handwriting was $20 \%$ faster if participants were able to manipulate the paper with their non-dominant hand, there is an opportunity to investigate if digital manipulations can lead to the same effect, or alternatively how hardware can be designed to invite for such manipulations with the ND-hand (e.g., continuing on [15]).

\section{Limitations and Suggestions for Future Studies}

There are limitations of our study, such as that the findings could have been affected by the lack of participants familiarity with digital tools, as noticeable in some of our findings that relate to lack of confidence and having to learn and practice in order to get better. The novelty and lack of confidence with the application could have increased the participant's cognitive load. For future work, a next study could investigate the differences between novice and expert usage and look into how users learn to adapt to digital tools, and the difference in attitudes before and after feeling competent at using it. Furthermore, a diary study recording the day-to-day use of digital and analogue tools for sketchnoting and note-taking could explore the context of usage of each platform.

\section{CONCLUSION}

Our study explored the differences in sketchnoting techniques and experiences when using analogue and digital tools. Results showed that participants found it harder to make sense of the available space in the digital. It was also 
found that although flexibility was praised as a key feature of digital tools, the simplicity found when sketchnoting on paper was preferred to the choice overload. In addition, although the texture of paper was preferred, participants concluded that it was only a matter of getting used to it. On top of that, participants reported feeling more confident when using paper, but claimed that digital tools were more convenient due to portability and format. In summary, these findings contribute to a better understanding of the unique possibilities of the two platforms.

\section{ACKNOWLEDGMENTS}

We would like to thank our participants for their motivation and willingness to collaborate in this study. The project leading to these results has received funding from "la Caixa" Foundation (ID 100010434).

\section{REFERENCES}

[1] Michelle Annett, Fraser Anderson, Walter F. Bischof, and Anoop Gupta. 2014. The Pen is Mightier: Understanding Stylus Behaviour While Inking on Tablets. In Proceedings of Graphics Interface 2014 (GI '14), 193-200.

[2] Michelle Annett, Anoop Gupta, and Walter F. Bischof. 2014. Exploring and Understanding Unintended Touch During Direct Pen Interaction. ACM Trans. Comput.Hum. Interact. 21, 5: 28:1-28:39. https://doi.org/10.1145/2674915

[3] Michelle Kathryn Annett. 2014. The Fundamental Issues of Pen-Based Interaction with Tablet Devices. $\mathrm{PhD}$ Dissertation, Department of Computer Science, University of Alberta.

[4] Rachel Bellamy, Michael Desmond, Jacquelyn Martino, Paul Matchen, Harold Ossher, John Richards, and Cal Swart. 2011. Sketching Tools for Ideation (NIER Track). In Proceedings of the 33rd International Conference on Software Engineering (ICSE '11), 808811. https://doi.org/10.1145/1985793.1985909

[5] Ann Blandford, Dominic Furniss, and Stephann Makri. 2016. Qualitative HCI Research: Going Behind the Scenes. Synthesis Lectures on Human-Centered Informatics 9, 1: 1-115. https://doi.org/10.2200/S00706ED1V01Y201602HCI03 4

[6] Svetlana Boym. 2012. Nostalgic Technology: Multitasking with Clouds. Photoworks. Retrieved May 21, 2019 from https://photoworks.org.uk/nostalgictechnology-multitasking-clouds/

[7] Peter Brandl, Christoph Richter, and Michael Haller. 2010. NiCEBook: Supporting Natural Note Taking. In Proceedings of the SIGCHI Conference on Human Factors in Computing Systems (CHI '10), 599-608. https://doi.org/10.1145/1753326.1753417

[8] Virginia Braun and Victoria Clarke. 2006. Using thematic analysis in psychology. Qualitative Research in Psychology 3, 2: 77-101. https://doi.org/10.1191/1478088706qp063oa
[9] Derek Bruff. 2014. Why Use Sketchnotes in the Classroom? - Agile Learning. Agile Learning. Retrieved April 11, 2019 from http://derekbruff.org/?p=2902

[10] J. M. Carroll. 1990. An overview of minimalist instruction. In Twenty-Third Annual Hawaii International Conference on System Sciences, 210-219 vol.4. https://doi.org/10.1109/HICSS.1990.205259

[11] Jara Dean-Coffey. 2013. Graphic Recording. New Directions for Evaluation 2013, 140: 47-67. https://doi.org/10.1002/ev.20073

[12] Robert Dimeo. 2016. Sketchnoting: An Analog Skill in the Digital Age. SIGCAS Comput. Soc. 46, 3: 9-16. https://doi.org/10.1145/3024949.3024951

[13] Veronica Erb. 2012. How to start sketchnoting. Bulletin of the American Society for Information Science and Technology 39, 1: 22-23. https://doi.org/10.1002/bult.2012.1720390108

[14] Mark Evans and Noor Aldoy. 2016. Digital Design Sketching using the Tablet PC. The Design Journal 19, 5: 763-787.

https://doi.org/10.1080/14606925.2016.1196091

[15] George Fitzmaurice, Thomas Baudel, Gordon Kurtenbach, and Bill Buxton. 1997. A GUI Paradigm Using Tablets, Two-hands and Transparency. In CHI '97 Extended Abstracts on Human Factors in Computing Systems (CHI EA '97), 212-213. https://doi.org/10.1145/1120212.1120348

[16] Sabrina Gerth, Thomas Dolk, Annegret Klassert, Michael Fliesser, Martin H. Fischer, Guido Nottbusch, and Julia Festman. 2016. Adapting to the surface: A comparison of handwriting measures when writing on a tablet computer and on paper. Human Movement Science 48: 62-73. https://doi.org/10.1016/j.humov.2016.04.006

[17] Sabrina Gerth, Annegret Klassert, Thomas Dolk, Michael Fliesser, Martin H. Fischer, Guido Nottbusch, and Julia Festman. 2016. Is Handwriting Performance Affected by the Writing Surface? Comparing Preschoolers', Second Graders', and Adults' Writing Performance on a Tablet vs. Paper. Frontiers in Psychology 7. https://doi.org/10.3389/fpsyg.2016.01308

[18] Christina Goulding. 2005. Grounded theory, ethnography and phenomenology. European Journal of Marketing. https://doi.org/10.1108/03090560510581782

[19] Saul Greenberg, Sheelagh Carpendale, Nicolai Marquardt, and Bill Buxton. 2012. Sketching User Experiences: The Workbook. Morgan Kaufmann.

[20] Yves Guiard. 1987. Asymmetric division of labor in human skilled bimanual action: the kinematic chain as a model. Journal of Motor Behavior 19, 4: 486-517. https://doi.org/10.1080/00222895.1987.10735426

[21] Ken Hinckley, Koji Yatani, Michel Pahud, Nicole Coddington, Jenny Rodenhouse, Andy Wilson, Hrvoje Benko, and Bill Buxton. 2010. Pen + Touch $=$ New Tools. In Proceedings of the 23Nd Annual ACM 
Symposium on User Interface Software and Technology (UIST '10), 27-36.

https://doi.org/10.1145/1866029.1866036

[22] Ken Hinckley, Koji Yatani, Michel Pahud, Nicole Coddington, Jenny Rodenhouse, Andy Wilson, Hrvoje Benko, and Bill Buxton. 2010. Manual Deskterity: An Exploration of Simultaneous Pen + Touch Direct Input. In CHI '10 Extended Abstracts on Human Factors in Computing Systems (CHI EA '10), 2793-2802. https://doi.org/10.1145/1753846.1753865

[23] Jonathan Lazar, Jinjuan Heidi Feng, and Harry Hochheiser. 2017. Research Methods in HumanComputer Interaction. Morgan Kaufmann.

[24] Yang Li, Ken Hinckley, Ken Hinckley, Zhiwei Guan, and James A. Landay. 2005. Experimental Analysis of Mode Switching Techniques in Pen-based User Interfaces. In Proceedings of the SIGCHI Conference on Human Factors in Computing Systems (CHI '05), 461470. https://doi.org/10.1145/1054972.1055036

[25] Iain Macdonald. 2014. Digital and Paper Sketchbooks: Learning New Ways of Drawing and Designing. TRACEY journal: Drawing and Visualisation Research. Retrieved from http://ualresearchonline.arts.ac.uk/10758/

[26] Penelope Mendonça. 2016. Graphic facilitation, sketchnoting, journalism and "The Doodle Revolution": New dimensions in comics scholarship. Studies in Comics 7, 1: 127-152. https://doi.org/10.1386/stic.7.1.127 1

[27] Pam A. Mueller and Daniel M. Oppenheimer. 2014. The Pen Is Mightier Than the Keyboard: Advantages of Longhand Over Laptop Note Taking. Psychological Science 25, 6: 1159-1168. https://doi.org/10.1177/0956797614524581

[28] V. Paepcke-Hjeltness, M. Mina, and A. Cyamani. 2017. Sketchnoting: A new approach to developing visual communication ability, improving critical thinking and creative confidence for engineering and design students. In 2017 IEEE Frontiers in Education Conference (FIE), 1-5. https://doi.org/10.1109/FIE.2017.8190659

[29] Verena Paepcke-Hjeltness, Janet Johnson, Luke Lefebvre, O. P. McCubbins, Holly Bender, Maren Wolff, Samantha Pritchard, and Foy Mills. 2018. Sketchnoting A Methodology: Fostering Team Based Learning Conversations. Team-Based Learning Presentations and Posters. Retrieved from https://lib.dr.iastate.edu/tbl_presentations/4

[30] Stefanie Panke. 2018. EdTech Research: Ready For Your Next Conference? Consider Sketchnoting to Learn and Connect. AACE. Retrieved April 11, 2019 from http://innovate.aace.org/review/edtech-research-gettingready-for-your-next-edtech-conference-considersketchnoting-to-learn-and-connect/

[31] Panagiotis Parthenios. 2005. Analog vs. Digital : why bother? In First International Conference on Critical
Digital: What Matter(s)? Harvard University Graduate School of Design, 117-128.

[32] Karin Perry and Holly Weimar. 2017. Sketchnoting: You and Your Students will Benefit. 1248-1255. Retrieved April 11, 2019 from https://www.learntechlib.org/primary/p/177901/

[33] Annie Piolat, Thierry Olive, and Ronald T. Kellogg. 2005. Cognitive effort during note taking. Applied Cognitive Psychology 19, 3: 291-312. https://doi.org/10.1002/acp.1086

[34] Yi Ren, Yang Li, and Edward Lank. 2014. InkAnchor: Enhancing Informal Ink-based Note Taking on Touchscreen Mobile Phones. In Proceedings of the SIGCHI Conference on Human Factors in Computing Systems (CHI '14), 1123-1132.

https://doi.org/10.1145/2556288.2557302

[35] Yann Riche, Nathalie Henry Riche, Ken Hinckley, Sheri Panabaker, Sarah Fuelling, and Sarah Williams. 2017. As We May Ink?: Learning from Everyday Analog Pen Use to Improve Digital Ink Experiences. In Proceedings of the 2017 CHI Conference on Human Factors in Computing Systems (CHI '17), 3241-3253. https://doi.org/10.1145/3025453.3025716

[36] Mike Rohde. 2013. The sketchnote handbook: the illustrated guide to visual note taking. Peachpit Press, San Francisco, CA.

[37] Barry Schwartz. 2005. The Paradox of Choice: Why More Is Less. Harper Perennial, New York, NY.

[38] Abigail J. Sellen and Richard H.R. Harper. 2003. The Myth of the Paperless Office. MIT Press, Cambridge, MA, USA.

[39] R. Neal Shambaugh. 1994. Personalized Meanings: The Cognitive Potentials of Visual Notetaking. In 1994 Eastern Educational Research Association. Retrieved September 19, 2019 from https://eric.ed.gov/?id=ED365969

[40] M. Shilman, Zile Wei, Sashi Raghupathy, P. Simard, and D. Jones. 2003. Discerning structure from freeform handwritten notes. In Seventh International Conference on Document Analysis and Recognition, 2003. Proceedings., 60-65 vol.1. https://doi.org/10.1109/ICDAR.2003.1227628

[41] Catherine Stones and Tom Cassidy. 2007. Comparing synthesis strategies of novice graphic designers using digital and traditional design tools. Design Studies 28, 1: 59-72. https://doi.org/10.1016/j.destud.2006.09.001

[42] Catherine Stones and Tom Cassidy. 2010. Seeing and discovering: how do student designers reinterpret sketches and digital marks during graphic design ideation? Design Studies 31, 5: 439-460. https://doi.org/10.1016/j.destud.2010.05.003

[43] Miriam Sturdee, Makayla Lewis, and Nicolai Marquardt. 2018. SketchBlog \#1: The Rise and Rise of the Sketchnote. Interactions 25, 6: 6-8. https://doi.org/10.1145/3281661 
[44] Miriam Sturdee, Makayla Lewis, and Nicolai Marquardt. 2018. Feeling SketCHI?: the lasting appeal of the drawn image in HCI. Interactions. Retrieved January 8, 2020 from https://dl.acm.org/doi/abs/10.1145/3274562

[45] Minghui Sun, Xiang Cao, Hyunyoung Song, Shahram Izadi, Hrvoje Benko, Francois Guimbretiere, Xiangshi Ren, and Ken Hinckley. 2011. Enhancing Naturalness of Pen-and-tablet Drawing Through Context Sensing. In Proceedings of the ACM International Conference on Interactive Tabletops and Surfaces (ITS '11), 83-86. https://doi.org/10.1145/2076354.2076371

[46] Lucia Terrenghi, David Kirk, Abigail Sellen, and Shahram Izadi. 2007. Affordances for Manipulation of
Physical Versus Digital Media on Interactive Surfaces. In Proceedings of the SIGCHI Conference on Human Factors in Computing Systems (CHI '07), 1157-1166. https://doi.org/10.1145/1240624.1240799

[47] Kirsten S. Traynor. 2015. Sketch Notes: Art that Engages Online. Bee World 92, 1: 23-25. https://doi.org/10.1080/0005772X.2015.1047638

[48] Theophanis Tsandilas, Magdalini Grammatikou, and Stéphane Huot. 2015. BricoSketch: Mixing Paper and Computer Drawing Tools in Professional Illustration. In Proceedings of the 2015 International Conference on Interactive Tabletops \& Surfaces (ITS '15), 127-136. https://doi.org/10.1145/2817721.2817729 